\title{
Becoming Responsible in a "Socially Seismic" Environment: Mental Health as a Marker of Community Recovery
}

Frederick M. Burkle Jr, MD, MPH, DTM, Judith Bass, PhD, MPH, and Paul Bolton, MBBS, MPH

I $\mathrm{n}$ this issue of Disaster Medicine and Public Health Preparedness, Abramson et $\mathrm{al}^{1}$ provide one more study exploring the importance of the mental health consequences of a disaster. The authors begin by discussing the nature of disasters and their mental health sequelae in developing countries, where severe and long-lasting consequences, such as large-scale destruction and displacement of largely poor populations, are not rare. Over time, disrupted "social vulnerabilities and inadequate infrastructure and lifelines" become common and long-lasting contributors to the psychological burden of the original catastrophic event. The echoes of Hurricane Katrina and its aftermath are clear. In terms of the size of the disaster, the inability to mount an effective response, and the subsequent displacement of mainly poor people, the United States has experienced and is continuing
... people are resilient when given the opportunity and resources to use their personal and communal coping systems. sonal and communal coping systems. When these resources and opportunities are not provided, populations become passively dependent on recovery services provided by others.

The similarities between Hurricane Katrina and disasters in developing countries likely extend beyond duration and complexity of the postacute phase and potential application of developing world experience to the Katrina response. The same challenges that we and other researchers and responders face in studying resulting mental health issues are clearly relevant to Abramson and colleagues' study. As with other postdisaster research among populations still subject to displacement and stress, there is uncertainty about the clinical significance of the study results. Abramson et al describe the multiple and pervasive stresses that continue to be experienced by the study population as a result of Katrina and its aftermath. However, they, and the tools they used in their study, are unable to disto experience something of comparable size, complexity, and duration to the complicated disasters that have occurred mostly in developing countries.

It is no surprise that many families affected by Katrina, still displaced and living in temporary housing and uncertain conditions, would continue, like their counterparts in developing countries, to experience symptoms of anxiety and depression. In retrospect, this also suggests that the experience of international organizations accustomed to working in developing countries, many of which are based in the United States, may have been relevant in organizing the postKatrina response. For example, if some of the standard approaches used in international disaster response had been followed in the immediate- and short-term period following the hurricane, including keeping families and communities together and recruiting the people affected in the planning and development of the recovery programs, some of the distress may have been mitigated. These standards are based in part on experience that suggests that people are resilient when given the opportunity and resources to use their per- tinguish between mental health effects and "normal" response to abnormal circumstances. The authors suggest that the increased prevalence and severity of symptoms (and syndromes, such as depression, anxiety, and conduct disorders) that they and others have found are mental health issues. Proceeding from this viewpoint, they consider even nonclinical interventions to restore social and psychological needs (as described by Silove and coworkers' adaptation and development after persecution and trauma [ADAPT] model) as "therapeutic." However, an alternative possibility exists in any situation in which stress, disruption, and shortages persist - that these symptoms and syndromes are normal responses among mentally healthy respondents.

In our own work in developing countries, we have repeatedly faced the same issue of distinguishing between disordered and "normal" responses to abnormal circumstances. Reliance on the Diagnostic and Statistical Manual of Mental Disorders (DSM) or International Classification of Diseases to describe and detect mental health problems often poses a problem 
because both systems are based largely on the assumption that a person's environment is stable and not overtly stressful. When this assumption does not hold, one is unable to use symptom assessments to distinguish between stress and disorder. Instruments based on the DSM and International Classification of Diseases therefore have the same problem. In a discussion of this issue, Horwitz ${ }^{2}$ notes that the DSM-IV does try to address this by requiring that a disorder meet the criterion of not being "merely an expectable and culturally sanctioned response to a particular event." Whereas this is noted in the introduction to the DSM, it is not explicitly repeated further under the diagnostic criteria for the various disorders. ${ }^{3}$ Our experience is that this criterion is not well respected when DSM-based instruments are used in situations of ongoing stress and disruption.

Recently we have begun to address this issue by emphasizing other diagnostic models to detect disorders. Taking our cue from the DSM, our primary approach has been to first explore what the local people we are studying consider to constitute "disorders." Assessment tools then emphasize the detection of these disorders as the basis for assessing mental health. This approach is based on the expectation that local people can distinguish, within their own culture and current situation, between a "normal" response to their abnormal circumstances and a decompensated response. Referring back to the study by Abramson et al, it would have been interesting to have asked the interviewees, particularly those exhibiting symptoms, whether they regarded themselves as responding appropriately to their situation. The fact that $93 \%$ of respondents felt that they could handle most things that were happening in their lives would seem to suggest a high percentage of people who feel that they are responding appropriately to their situation.

The distinction between disordered and "normal" responses to a stressful situation has programmatic relevance. Specific mental health care, including medical and psychological care, is required in cases in which the symptoms and accompanying dysfunction represent disorder. However, where symptoms are a normal response to a stressful situation, it is more appropriate to provide general services to improve the situation, including speeding up the system of providing appropriate housing, improving community safety and services, offering safe and effective child care and educational opportunities, and helping affected populations find gainful employment. Studies that cannot distinguish between disordered and normal response are unable to suggest which combination of mental health services and more general services is appropriate.

Clearly, as the authors state, a "socially seismic" environment is provoked by such compelling events. We can improve our planning and responses by understanding the culture and point of view of the population being served, learning how to focus on both mental distress as well as disorder when it is appropriate, and in adopting what has been learned in situations outside the United States. We can better develop an appropriate model for response to and recovery from future disasters that may occur within our own borders.

\section{About the Authors}

Dr Burkle is a senior fellow at the Harvard Humanitarian Initiative, Harvard University; Dr Bass is an assistant professor in the Department of Mental Health, Johns Hopkins Bloomberg School of Public Health; and Dr Bolton is an associate scientist in the Center for Refugee and Disaster Response, Johns Hopkins Bloomberg School of Public Health.

Address correspondence and reprint requests to Dr Frederick M. Burkle Jr, Harvard Humanitarian Initiative, Harvard University, 14 Story St, 2nd Floor, Cambridge, MA 02138 (e-mail: skipmd77@aol.com).

Received and accepted for publication March 12, 2008.

ISSN: 1935-7893 (C) 2008 by the American Medical Association and Lippincott Williams \& Wilkins.

DOI: $10.1097 / D M P .0 b 013 \mathrm{e} 3181739 \mathrm{fe} 1$

Authors' Disclosures

The authors report no conflicts of interest.

\section{REFERENCES}

1. Abramson D, Stehling-Ariza T, Garfield R, Redlener I. The prevalence and predictors of mental health distress post-Katrina: findings from the Gulf Coast Child and Family Health Study. Disaster Med Public Health Preparedness. 2008;2:77-86.

2. Horwitz AV. Distinguishing distress from disorder as psychological outcomes of stressful social arrangements. Health. 2007;11:273-289.

3. American Psychiatric Association. Diagnostic and Statistical Manual of Mental Disorders, 4th ed. Washington, DC: American Psychiatric Association; 2000. 